\title{
Central Localization of Gustatory Perception: An Experimental Study
}

\author{
WESLEY H. BRADLEY ${ }^{1}$ \\ Department of Otolaryngology, University of Michigan Medical School, \\ Ann Arbor, Michigan
}

There appears to be rather general agreement as to the structure of the peripheral endings involved, as well as the course and the anatomical variations to be found in the root fibers of the VII, IX, and X cranial nerves which carry impulses set up by gustatory stimuli. The fibers of these gustatory components on entering the brain separate from the other components of the respective nerves to become associated with the fasciculus solitarius and to terminate in its associated gray, the dorsal visceral gray. There is considerable evidence that, from this dorsal visceral gray, there is a discharge forward over bundles accompanying the medial lemniscus to the contralateral hypothalamus and dorsal thalamus (Allen, '23a, '23b; Walker, '38). Those fibers passing to the hypothalamus separate from the medial lemniscus at midbrain levels to proceed to their destination by way of the mammillary peduncle. The possibility of a discharge to the homolateral thalamus and hypothalamus has never been proved or disproved.

Less information exists with regard to the thalamic and cortical centers concerned with taste. The uncertainty as to the location of cortical centers for gustatory impulses may be explained on the basis of: (1) the difficulty involved in accurate experimental and clinical testing for taste; and (2) the infrequency of clinical conditions in which a central lesion involving taste is seen at a time when selective testing is possible. The following experimental study was undertaken in an attempt to explore further the thalamic and cortical centers for gustatory sensation.
REVIEW OF THE LITERATURE

Previous authors (Ferrier, 1886; Herzog, '28; Marcus, '34) have concluded that all the cortical areas concerned with representation for gustatory sensation are in close relationship to the cortical olfactory areas. Börnstein ('40a) discussed three possibilities of gustatory cortical localization: e.g., that the centers are in the temporal lobe, the insula, or the parietal operculum. He felt that the functional relation taste bears to tactile sensation predicates a convergence of these two kinds of sensation in the cortex rather than one of gustatory and olfactory sensations. He, therefore, favored the parietal operculum as the probable location for cortical representation. In a subsequent report, Börnstein ('40b) described a technique for semiquantitative testing of taste and presented the case histories of three patients who had suffered bullet wounds of the parietal bone which produced lesions of the pre- and the post-central gyri of the left hemisphere. Testing, which was done four to six years after the accident, revealed changes in taste perception on the right side of the tongue in all three patients. These changes varied from ageusia for all modalities in one patient, through ageusia for sweet and sour and hypoageusia for salty and bitter in a second patient, to hypoageusia for all modalities in the third patient. Börnstein suggested these case reports provided further support for the theory that the parietal operculum is the cortical area concerned with taste.

1 Present address: 1100 East Genesee Street, Syracuse 10, New York. 
Shenkin and Lewey ('43) reported an interesting case history of a 27 year old man with the complaint of headache and generalized convulsive seizures. The seizures were preceded by an aura of a sour to bitter taste. Testing demonstrated that the perception of sweetness was absent over the left side of the tongue, but bitter and salty substances were recognized over the entire tongue. Surgical exposure revealed a convergence of engorged dilated vessels located in the Sylvian fissure. The point of greatest dilatation of the vessels and of underlying compression was over the lowest portion of the postcentral gyrus. Gustatory hallucinations were obtained by Foerster ('36) in man following unilateral damage to the inferior part of the postcentral convolution; unilateral removal of the area did not result in ageusia.

Penfield and Rasmussen ('50) record the production of a sensation of taste by stimulation of the parietal operculum that joins the upper bank to the insular cortex. They also mentioned one area on the floor of the insula which when stimulated produced a curiously disagreeable taste.

Adler ('34) presented the case history of a patient who had lost taste sensation on one side of the tongue and all sensation on the same side of the face. Post-mortem examination of the brain revealed a thalamic tumor close to the ventricle and involving the medial part of the arcuate nucleus and the centromedial nucleus. Adler emphasized the infrequency of the occurrence of such cases which allow clinical testing, for the most common pathology in this area is hemorrhage with subsequent coma and death. Walker ('38) concluded that evidence suggested there is a tract connecting the medullary nuclei concerned in gustatory sensation with the medial part of the ventral nucleus of the thalamus in the region of the nucleus centrum medianum. Blum, Walker, and Ruch ('43), from a study of stereotaxic thalamic lesions in the monkey, regarded the arcuate nucleus (nucleus ventralis posteromedialis) as the thalamic area concerned with taste. They thought that taste and somatic sensation are contiguously represented in the thalamus. They also sug- gested that Broadman's areas 3 and 1 at the foot of the precentral gyrus represent the cortical locus of taste. A continuation of the preceding study by Patton, Ruch, and Walker ('44) suggested that the severity of the taste defect paralleled the amount of damage to the nucleus ventralis posteromedialis (around the nucleus). The same authors state that taste is also represented cortically in the post-central gyrus.

Studies on taste responses in the cat after midbrain transection were reported by Macht ('51). The level of transection dorsally was at the rostral border of the colliculi, and ventrally varied from just behind the mammillary bodies to the rostral border of the pons. Macht found that sweet solutions were never rejected by any of the test animals regardless of the concentration, but that bitter, salt, and sour solutions were all rejected by all animals at certain concentrations. The thresholds for rejection were found to be about the same as those at which normal cats also rejected similar test materials. Macht concluded that certain discriminatory responses relating to taste have a subcortical localization in the cat.

Schwartz, Roulhac, Lam, and O'Leary (51) presented the findings in an interesting clinical case. Division of the sensory roots of V, VII, IX, and the rostral roots of $X$, as well as first, second, and third cervical dorsal roots, was performed unilaterally in a patient for the relief of intractable pain. Death ensued 116 days after surgery, and the brain stem was prepared for Marchi technique. Selected sections were stained by the Nissl, Weigert, and Bodian techniques. Contributions of VII, IX, and $X$ nerves to the fasciculus solitarius were demonstrated, and some evidence was obtained supporting the existence of gustatory nuclei related to VII and IX.

Portions of the frontal and the temporal opercula, an area across the insula, and an area in the temporal cortex were all regarded as related to gustatory functions in the monkey by Bagshaw and Pribram (53). Benjamin and Akert ('59) identified, as the gustatory cortex in the rat, a cortical strip dorsal to the rhinal fissure in somatic 
sensory area I. Ablation of this strip bilaterally was found to produce a partial taste deficit. Ablation of the fringe area as well interfered seriously with taste discrimination.

\section{MATERIALS AND METHODS}

In this study, the monkey, Macaca mulatta, was the animal selected for testing. Only one modality, bitter, was used for testing taste. Initially a $0.5 \%$ solution of quinine sulfate was employed. However, a preliminary survey revealed that this solution was inadequate, and quinine powder was therefore used throughout the remainder of the study.

The animals were all initially tested after food had been withheld for at least a 12-hour period. A food known to be readily accepted, e.g., banana, was then offered. The fast was then repeated, the first food then offered was banana coated with quinine powder. Prior to surgery, all the animals tasted and discarded the quinine treated banana, regardless of the length of their fast. However, as soon as the untreated banana was offered and tasted, it was readily accepted and consumed.

After testing each animal as described, surgery under sterile technique was carried out. The skull was trephined and the area to be destroyed was exposed and obliterated by cauterization or removed by suction. In two cases a more discrete lesion was placed with an insulated electrode passed through a trephine opening in the skull. In these cases a stereotaxic instrument was used to maintain the animal and the needle electrode in position. The settings for the stereotaxic instrument were based on the figures of Atlas and Ingram ('37).

Postoperatively, the animals were again tested as soon as they were well enough to fast, using first the quinine treated banana and then the untreated banana. Each animal was then sacrificed and the carcass perfused. The brain was removed in order to check the positions of the lesions. The brains of the two animals in which stereotaxic lesions were placed were prepared by the Marchi technique and the sections checked to determine the site of the lesions.
RESULTS

TABLE 1

\begin{tabular}{|c|c|}
\hline $\begin{array}{l}\text { Animal } \\
\text { number }\end{array}$ & Type of lesions produced \\
\hline 1 & Bilateral precentral gyrus lesions \\
\hline 2 & Bilateral precentral gyrus lesions \\
\hline \multirow[t]{2}{*}{3} & Bilateral section of the olfactory bulbs \\
\hline & $\begin{array}{l}\text { Bilateral precentral gyrus lesions ex- } \\
\text { tending into postcentral gyrus }\end{array}$ \\
\hline 4 & Bilateral insula lesions \\
\hline 5 & Bilateral hippocampal gyrus lesions \\
\hline 6 & Bilateral extensive removal of cortex \\
\hline 7 & Bilateral stereotaxic (thalamic) lesions \\
\hline \multirow[t]{2}{*}{8} & Bilateral section of the olfactory bulbs \\
\hline & Bilateral stereotaxic (thalamic) lesions \\
\hline 9 & Bilateral superior collicular lesions \\
\hline
\end{tabular}

\section{Monkey 1}

Male. Weight - $1.8 \mathrm{~kg}$. The animal consistently rejected a $0.5 \%$ quinine sulfate solution, even after a 24-hour period without food or water. After the final preoperative testing a left craniotomy was performed and a lesion was placed in the lower end of the precentral gyrus. The site of the lesion was just below the area of cortex where stimulation produced movement of the right side of the face and very near the area for tongue responses. The lesion, produced by cautery, was approximately $8 \times 10 \mathrm{~mm}$ with a depth of approximately $3 \mathrm{~mm}$. When tested postoperatively the animal still rejected the quinine solution. A right craniotomy was then performed and a similar lesion was made on the right side. Postoperative testing revealed that the monkey still rejected the quinine solution. The postmortem examination of the brain confirmed the positions of the lesions (fig. 1 ).

\section{Monkey 2}

Male. Weight - $2 \mathrm{~kg}$. The animal consistently rejected the bitter solution $(0.5 \%$ quinine sulfate). A left craniotomy was performed and a lesion placed at the lower end of the precentral gyrus and extending into the lateral sulcus. The lesion, approximately $8 \times 10 \mathrm{~mm}$ and $3-4 \mathrm{~mm}$ deep, was made with a cautery. When tested post-operatively, the animal still rejected the bitter solution. A right craniotomy was then performed and a similar lesion was placed in the right precentral gyrus. When tested post-operatively, the animal still re- 


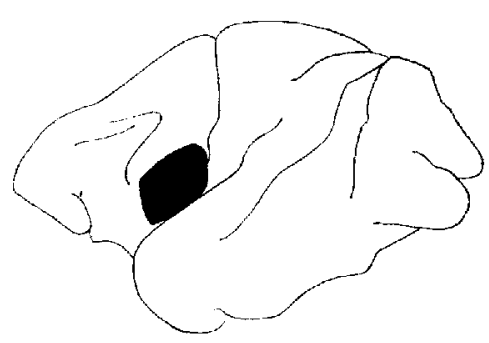

Monkey No.I

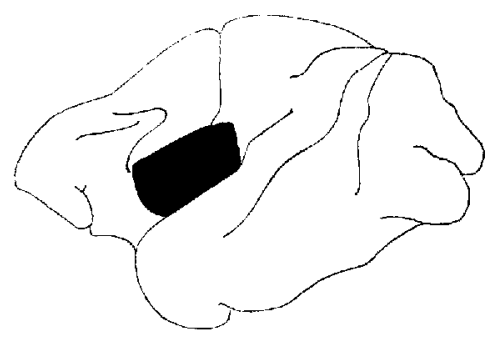

Monkey No. 3

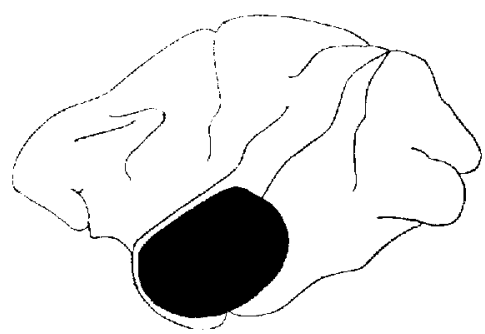

Monkey No. 5

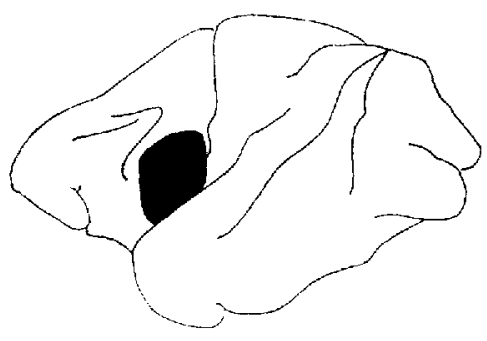

Monkey No. 2

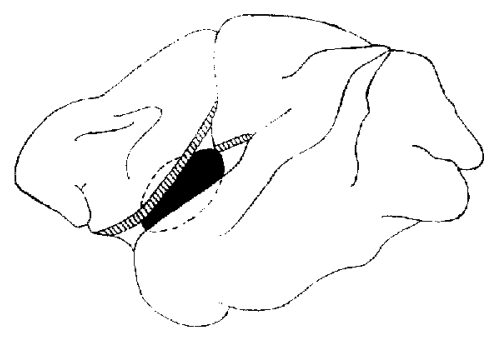

Monkey No. 4

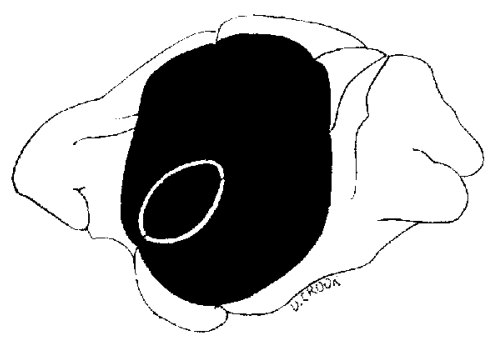

Monkey No. 6

Fig. 1 Outline drawings of the macaque brain showing the locations of the lesions in monkeys 1-6.

fused to drink the quinine solution. The postmortem examination confirmed the positions of the lesions (fig. 1).

\section{Monkey 3}

Female. Weight $-1.5 \mathrm{~kg}$. On repeated testing, the animal consistently rejected the bitter solution ( $0.5 \%$ quinine solution). A wide frontal craniotomy was performed and both olfactory bulbs were elevated and severed. When tested post-operatively, the animal still would not drink the quinine solution. Bilateral craniotomy was then performed and lesions placed bilaterally 
with a cautery. The lesions were approximately $8 \times 10 \mathrm{~mm}$ and $3 \mathrm{~mm}$ deep. They were located at the lower end of the precentral gyrus, involving the precentral gyrus, the area adjacent to it rostrally, and the postcentral gyrus and extended on to the opercular surface of these gyri. Postoperative testing showed that the animal would accept and drink the quinine solution, but portions of banana on which quinine powder had been sprinkled were discarded. However, the animal ate unusual things for a monkey; e.g. meat and cork. The postmortem examination confirmed the positions of the lesions (fig. 1).

\section{Monkey 4}

Male. Weight - $2 \mathrm{~kg}$. The monkey consistently rejected quinine-treated banana. A left temporo-parietal trephine was done and the lateral surface of the hemisphere exposed. The opercula were retracted and the island visualized and destroyed with the cautery. The middle cerebral artery was preserved. When tested postoperatively the animal still rejected the quinine-treated banana. A right temporo-parietal trephine was done and similar destruction of the right island achieved. Again the middle cerebral artery was preserved. Tested postoperatively the monkey still discarded the quinine-treated banana. The positions of the lesions were confirmed on postmortem examination (fig. 1).

\section{Monkey 5}

Male. Weight - $2.2 \mathrm{~kg}$. The monkey consistently rejected quinine-treated banana. A left temporo-parietal trephine was performed, the lateral wall of the temporal lobe removed with suction, and the lateral ventricle entered. The portion of the temporal gyrus in the tip of the temporal lobe, including the uncus and the hippocampal gyrus, was destroyed by cauterization. When tested postoperatively the animal still rejected quinine-treated banana. A right temporo-parietal trephine was performed and a similar lesion created. Tested postoperatively the monkey still discarded the quinine-treated banana. The positions of the lesions were confirmed on postmortem examination (fig. 1).

\section{Monkey 6}

Male. Weight $-2.4 \mathrm{~kg}$. The animal consistently rejected quinine-treated banana. Bilateral temporo-parietal trephines were done and these were enlarged to expose as much cortex as possible, bilaterally. Through these openings, extensive removal of the cortex was carried out bilaterally using suction. When tested postoperatively the animal still rejected the quinine-treated banana. The positions of the lesions were confirmed on postmortem examination (fig. 1).

\section{Monkey 7}

Male. Weight - $2.2 \mathrm{~kg}$. The animal consistently rejected quinine-treated banana. A left parietal trephine was performed, and a thalamic lesion was placed in the medial part of the nucleus ventralis posterior, using the stereotaxic apparatus. When tested postoperatively the animal still rejected the quinine-treated banana. A similar lesion was placed on the right side using the same technique. Tested postoperatively the monkey still discarded the quinine-treated banana.

On examination of the microscopic material the lesions were found to be in the medial portion of the medial lemniscus (where the secondary ascending gustatory tract is believed to be located). Figure 2, taken just rostral to the lesion, shows the degenerated fibers entering the most medial part of each nucleus ventralis posterior.

\section{Monkey 8}

Male. Weight - $2.7 \mathrm{~kg}$. The animal consistently discarded the quinine-treated banana. A wide frontal craniotomy was performed and both alfactory bulbs were elevated and severed. When tested postoperatively the animal still rejected the quinine-treated banana. A left parietal trephine was performed, and a thalamic lesion was placed in the medial part of the nucleus ventralis posterior, using the stereotaxic apparatus. Tested postoperatively the animal still discarded the quinine-treated banana. A similar lesion was then placed on the right side using the same technique. Tested postoperatively the animal still refused the quinine-treated banana. The positions of the lesions were 

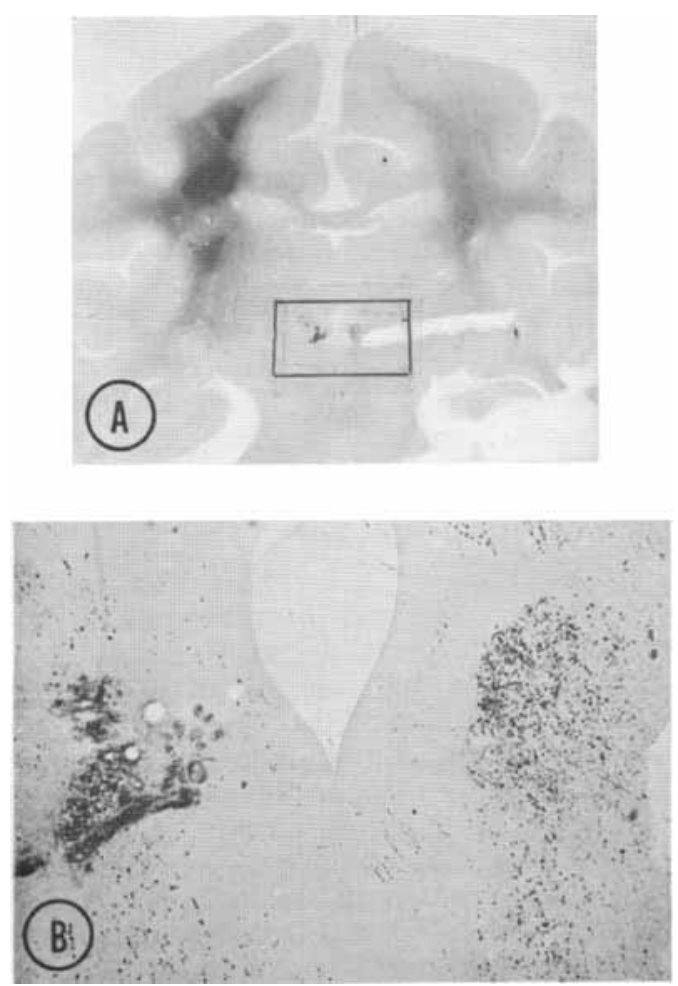

Fig, 2 A, a low power photomicrograph $(\times 1.7)$ and $B$, a high powcr photomicrograph $(\times 22)$ of the region indicated in $A$, from the brain of monkey 7. These pictures are rostral to the sites of the lesions. Each lesion was placed in the region where the most medial part of the medial lemniscus (the secondary ascending gustatory tract) was entering the nucleus ventralis posterior. The degenerated fascicles of the destroyed bundle are seen within the pars medialis of the nucleus.

confirmed on examination of the histologic section and were essentially the same as for monkey 7 (sce fig. 2).

\section{Monkey 9}

Male. Weight - $2 \mathrm{~kg}$. The animal consistently rejected quinine-treated banana. A left parieto-occipital trephine was made, and widened to expose the left occipital lobe and the caudal end of the parietal pole, including areas 18 and 19 . These areas were removed with suction and the superior colliculi were exposed, and destroyed bilaterally with cautery. When tested postoperatively the animal showed a marked indifference to all food, even bananas. He seemed to be slow in reacting to the quinine-treated banana, but after chewing it for six to ten seconds, he would discard it. The position of the lesion was confirmed on postmortem examination.

\section{DISCUSSION AND SUMMARY}

All monkeys with unilateral or bilateral lesions of the cortex reacted to the quinine. There was more slowing in response when an area was destroyed at the base of the central fissure involving the bordering portions of the precentral and postcentral gyri, including their opercular extensions (monkey 3 ). When the lesion was in this region, the monkey showed a decrease of discriminatory taste, possibly due to some loss of both gustatory and tactile modalities. Consequently, the monkey did not discriminate readily between edible and non-edible objects, although he still recognized a bitter taste.

With lesions in the medial part of the nucleus ventralis posterior of the dorsal thalamus or the medial lemniscus fibers to it, there was a slowing in the gustatory responses, but the animal still rejected the quinine. It will be remembered that Adler's (34) patient with a thalamic lesion in approximately the area represented by our lesion lost all types of taste sensibility except possibly a little bitter. With tectal and adjoining subtectal regions involved, the responses were definitely greatly reduced. The animal actually chewed the banana with the quinine for some seconds before rejecting it, and rejected it hesitantly. It should be emphasized again that in this instance, the lesion was bilateral.

From the foregoing results, it would appear that there is some midbrain appreciation of gustatory stimuli of the bitter type. This is sufficient in amount to lead to the rejection of bitter substances even if thalamic and cortical areas believed to be related to gustatory paths are destroyed. Such findings might be expected and therefore, explained phylogenetically. In the more primitive midbrain, the gustatory functions appear to be those concerned in general with body well-being or protection; in the phylogenetically later developed cortical areas the more delicate discriminatory gustatory functions are added. In establishing gustatory tests to evaluate brain 
involvements at various levels, it is essential to distinguish between tests for discriminatory as contrasted with tests for more gross types of gustatory sensibility.

Further study in this area is most obviously necessary, including both laboratory research and clinical observation. In the latter instance, careful testing and accurate recording of the data for correlation with surgical or autopsy findings is essential. Only through such a program and the reporting of our findings can our knowledge of the central gustatory areas and connections be improved.

Finally subjective gustatory sensations may be accompaniments of emotional states. The effects of gustatory stimulations may be retained in association areas of the hemispheres years after the initial stimulus and reappear when the same cortical region or an associated region becomes activated during emotional stress or by irritative lesions. Tumors in the region of the uncus and the hippocampal gyrus sometimes produce gustatory as well as olfactory aura.

\section{ACKNOWLEDGMENT}

The author acknowledges with thanks the help of Dr. Elizabeth C. Crosby in this study.

\section{LITERATURE CITED}

Adler, A. 1934 Zur Topik des Verlaufes der Geschmackssinnsfasern und anderer afferenter Bahnen im Thalamus. Ztschr. f. d. ges. Neurol. u. Psychiat, 149: 208-220.

Allen, W. F. 1923a Origin and distribution of the tractus solitarius in the guinea pig. J. Comp. Neur,, 35: 171-204.

$1923 \mathrm{~b}$ Origin and distribution of the secondary visceral fibers in the guinea pig. J. Comp. Neur., 35: 275-311.

Atlas, D., and W. R. Ingram 1937 Topography of the brain stem of the rhesus monkey with special reference to the diencephalon. J. Comp. Neur., 66: 263-289.

Bagshaw, M. H., and K. H. Pribram 1953 Cortical organization in gustation (Macaca mulatta). J. Neurophysiol., 16: 499-508.

Benjamin, R. M., and K. Akert 1959 Cortical and thalamic areas involved in taste discrimination in the albino rat. J. Comp. Neur., 111: 231-259.

Blum, M., A. E. Walker and T. C. Ruch 1943 Localization of taste in the thalamus of Macaca mulatta. Yale J. Biol. and Med, 16: 175-192.

Börnstein, W. S. 1940a Cortical representation of taste in man and monkey. I. Functional and anatomical relations of taste, olfaction, and somatic sensibility. Yale J. Biol. and Med., 12: $719-736$.

- 1940b Cortical representation of taste in man and monkey. II. The localization of the cortical taste area in man and a method of measuring impairment of taste in man. Yale J. Biol. and Med., 13: 133-156.

Ferrier, D. 1886 Functions of the Brain. G. P. Putnam's Sons, New York, 498 pp. and XXIII.

Foerster, O. 1936 Sensible cortical Felder. In: Handbuch der Neurologie, edited by 0 . Bumke and $O$. Foerster. J. Springer, Berlin, 6: 358-448.

Herzog, F, 1928 Uber die corticalen Zentren des Geschmacks und die Geruchs auf Grund von Beobachtungen bei Hypophysentumoren. Deutsche Ztschr. f. Nervenh,, 102: 221-238.

Macht, M. B. 1951 Subcortical localization of certain "taste" responses in the cat. Fed. Proc., 10: 88 .

Marcus, H. 1934 Die lokalisation des Geschmackszentrum. Acta psychiat. el neurol., 9: 85-116.

Patton, H. D., T. C. Ruch and A. E. Walker 1944 Experimental hypoguesia from Horsley-Clarke lesions of the thalamus in Macaca mulatta. J. Neurophysiol., 7: 171-184.

Penfield, W., and T. Rassmussen 1950 The Cerebral Cortex of Man. The Macmillan Company, New York, pp. 121, 248.

Schwartz, H. G., G. E. Roulhac, R. L. Lam and J. L. O'Leary 1951 Organization of the fasciculus solitarius in man. J. Comp. Neur., 94: 221-239.

Shenkin, H. A., and F. H. Lewey 1943 Aura of taste preceding convulsions associated with a lesion of the parietal operculum: Report of a case. Anat. neurol. and psychiat., 50:375-378.

Walker, A. E. 1938 The Primate Thalamus. University of Chicago Press, Chicago, p. 321. 